\title{
Nucleon Structure and Soft QCD from LHCb
}

\section{Agnieszka Oblakowska-Mucha ${ }^{a, 1, *}$ and Saliha Bashir ${ }^{a}$}

${ }^{a}$ Faculty of Physics and Applied Computer Science, AGH University of Science and Technology

Al. Mickiewicza 30, 30-059 Krakow, Poland

E-mail: Agnieszka.Mucha@agh.edu.pl

Since the LHC reached the TeV scale in the high-energy hadron collisions, understanding of soft processes becomes inevitable for the constraining of the high density regime of QCD. The measurement of the differential cross-section of prompt inclusive production of long-lived charged particles in proton-proton collisions at $\sqrt{s}=13 \mathrm{TeV}$ is performed by the LHCb experiment. The cross-section is evaluated as a function of transverse momentum and pseudorapidity and compared with the physics model used in current predictions. Comparison of the proton-proton and proton-lead collisions provides insights into the structure of the initial state. LHCb reports the determination of the nuclear modification factor at previously unexplored parton momentum fraction.

The Ninth Annual Conference on Large Hadron Collider Physics - LHCP2021

7-12 June 2021

Online

${ }^{1}$ for the LHCb Collaboration

*Speaker 


\section{Introduction}

The interaction between high-energy protons involves a wide spectrum of processes highly dependent on the fraction of the proton's momenta carried by the partons and the momentum transfer between partons. Each hard interaction between partons from two protons is usually accompanied by the soft interaction which comprises interactions between beam remnants and radiation of gluons in the initial and final state. All mentioned processes give rise to the particles with a low transverse momentum $p_{T}$. Therefore one can assume that proton-proton $(p p)$ collisions, even at high energy, are dominated by soft processes. The soft processes cannot be calculated from the first principles in perturbative quantum chromodynamics (QCD) and prediction are based on effective phenomenological models. Precision measurement of prompt long-lived charged particles production at the $\mathrm{TeV}$ scale allows to validate these models.

The LHCb experiment has been designed as forward spectrometer and equipped with the precise tracking stations and an efficient charged-hadron identification system [1]. Therefore it enables the studies in the forward pseudorapidity region, which is of a special interest for the LHC community and for cosmic-ray research as well. Modification of charge particle production rate in proton-ion $(p \mathrm{~Pb})$ collision in comparison to proton-proton collisions describes dynamics of Cold Nuclear Matter (CNM) effects [2].

\section{Measurement of prompt charged-particle production in proton-proton collisions at $\sqrt{s}=\mathbf{1 3} \mathrm{TeV}$}

The differential cross-section was measured at the LHCb experiment using a data sample collected with an unbiased trigger as a function of transverse momentum and pseudorapidity in the ranges $p_{T} \in[0.08,10) \mathrm{GeV} / \mathrm{c}$ and $\eta \in[2.0,4.8)$ [3]. The data was recorded at a centre-of-mass energy $\sqrt{s}=13 \mathrm{TeV}$ in 2015 and corresponded to an integrated luminosity of $5.4 \mathrm{nb}^{-1}$.

Prompt long-lived charged particles reconstructed as tracks which traversed the entire tracking system were considered in this measurement. The main background contribution came from fake tracks. Neural-network-based algorithms were used to reduce the tracks with high fake-track probability. The selection efficiency and remaining background, predominantly containing secondary particles, were modelled based on simulation with the correction from control measurements.

The original efficiencies evaluated for each particle type as a function of $p_{T}$ in two most forward $\eta$ bins are presented in Figure 1. The final efficiency was computed by summing the original efficiency for all particle classes multiplied by the corrected fraction. Combined correction reduced the original distribution up to $5 \%$. The total uncertainty of this evaluation varies from $0.9 \%$ to $5.1 \%$.

The measured differential cross-section of prompt inclusive production of long-lived charged particles is shown in Figure 2. It is compared to the predictions from the hadronic-interaction model. The differences occur in the range from $-26 \%$ to $+170 \%$, with the highest deviations observed for high- $p_{T}$ particles. The smallest deviations are observed for EPOS-LHC [4]. In comparison of the ratio of the differential cross-section for positively and negatively charged particles (Figure 2 right), the best description is given by Pythia 8.303 with the default Monash tune [6, 7] . 

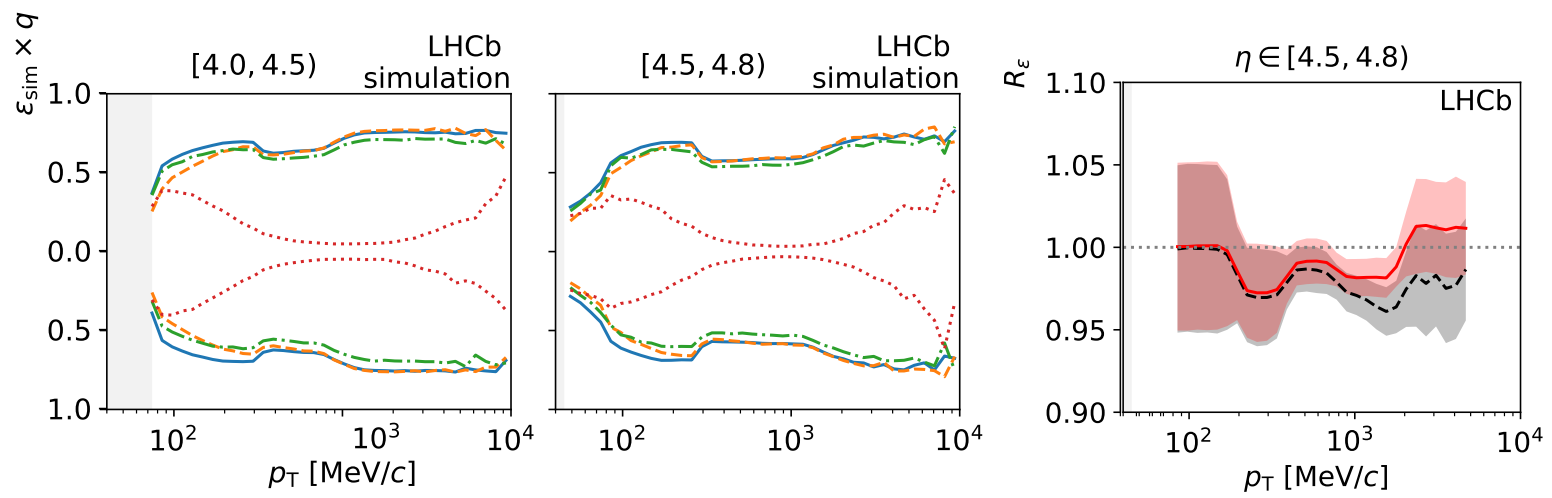

Figure 1: Efficiencies for different particle types in two intervals of $\eta$ and as a function of $p_{T}$ for the simulated sample (left and center plots). The product of the efficiency and the particle charge in units of the elementary charge is shown to separate values for oppositely charged particles The light-grey areas indicate the limit of the kinematic acceptance. Combined correction $R_{\epsilon}$ to the efficiency simulation for $\eta \in[4.5,4.8)$ (right plot). The bands indicate the systematic uncertainty.
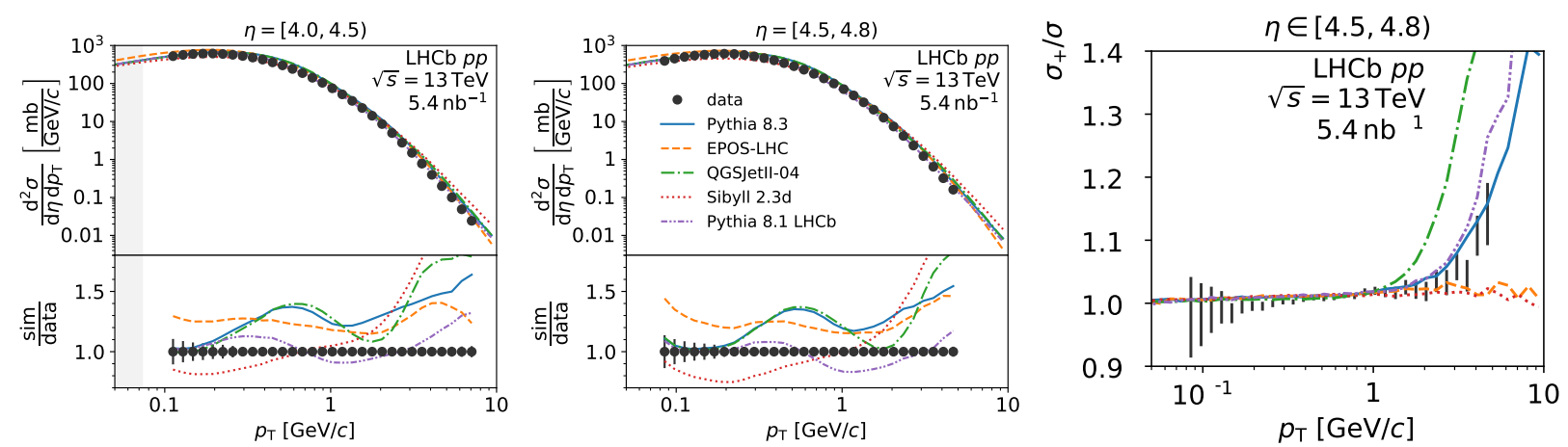

Figure 2: Differential cross-section of inclusive production of prompt long-lived charged particles in two intervals of $\eta$ as a function of $p_{T}$ (left and center plots). The error bars indicate the total uncertainty. The ratios of the model predictions and this measurement are shown in the lower subplots. Ratio of the differential cross-section for positively and negatively charged particles $\eta \in[4.5,4.8)$ (right plot). The lines labelled as Pythia 8.1 LHCb correspond to the weighted simulated samples of this analysis.

\section{Measurement of the nuclear modification factor and prompt charged particle production in $p \mathbf{P b}$ and $p p$ collisions at $\sqrt{s_{N N}}=5 \mathrm{TeV}$}

Comparison of charged particle production in $p p$ and $p \mathrm{~Pb}$ collisions gives a valuable insight into the initial-state nuclear interaction usually expressed by the nuclear modification factor $R_{p \mathrm{~Pb}}$. This parameter is defined as a ratio of the differential cross-sections in $p \mathrm{~Pb}$ and $p p$ collisions scaled with the ion mass number.

Two data samples were studied in the LHCb experiment: $p$ Pb collisions at $\sqrt{s_{N N}}=5 \mathrm{TeV}$ in the year 2013 and $p p$ data recorded in the year 2015 at the same centre-of-mass energy. The integrated luminosity considered for the $p \mathrm{~Pb}$ forward and backward configuration was $42.73 \pm 0.98$ $\mu \mathrm{b}^{-1}$ and $38.71 \pm 0.97 \mu \mathrm{b}^{-1}$ respectively. For the $p p$ data the recorded luminosity was $3.49 \pm 0.07$ $n b^{-1}$. 


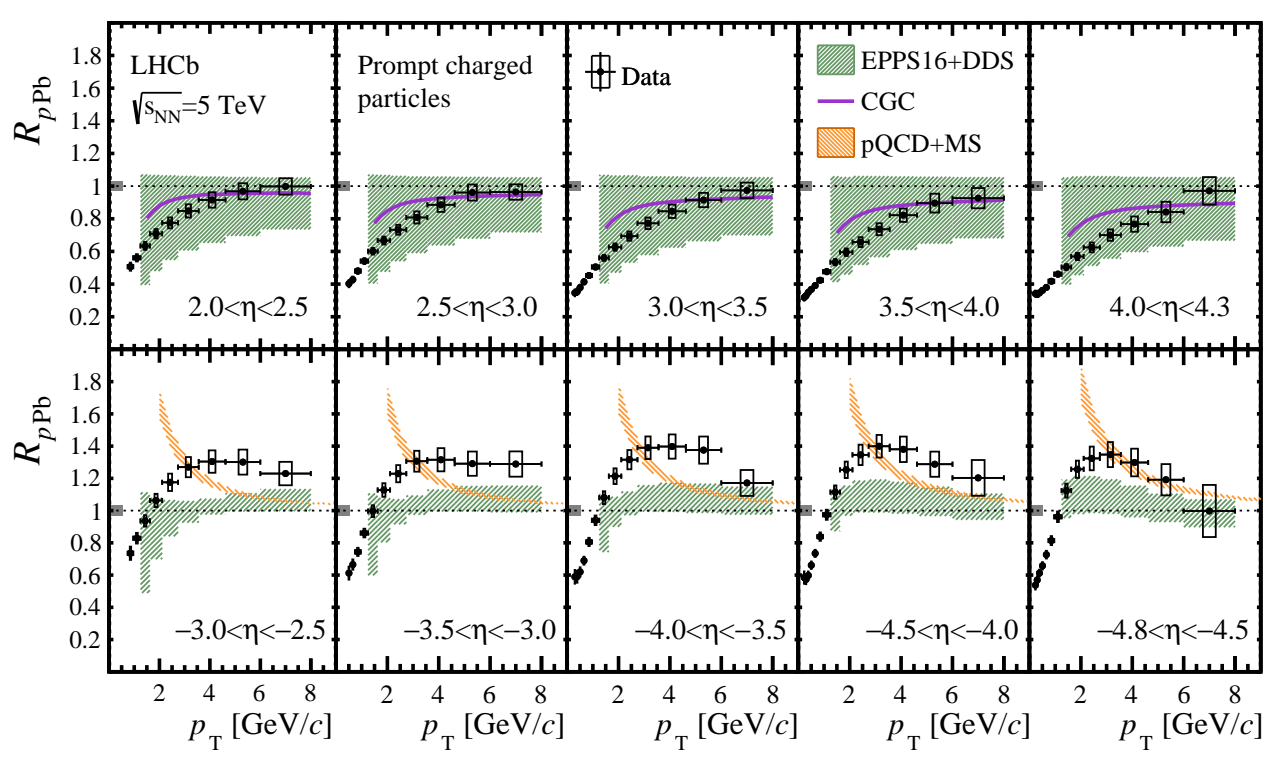

Figure 3: Nuclear modification factor as a function of $p_{T}$ in different $\eta$ intervals for the (top) forward and (bottom) backward regions, compared with the predictions from different models. Vertical error bars correspond to statistical uncertainties, open boxes to uncorrelated systematic uncertainty and the filled box at $R_{p \mathrm{~Pb}}=1$ to the correlated uncertainty from the luminosity.

Prompt charged particles were reconstructed from tracks with hits at the Vertex Detector and tracking stations after the magnet. Particles with momentum below $2 \mathrm{GeV} / \mathrm{c}$ were beyond the LHCb acceptance. Contribution from fake tracks were reduced with a neural-net based algorithm. Prompt charged particle yield was obtained after the corrections which accounted for the purity, reconstruction and selection efficiency, with the input from real data.

The LHCb results for the $R_{p \mathrm{~Pb}}$ in different $\left(\eta, p_{T}\right)$ bins are shown in Figure 3. In the forward region, the suppression of charged particle production in $p \mathrm{~Pb}$ collision relative to the $p p$ collision increases towards forward $\eta$. In the backward region the behaviour is different: the enhancement is visible in $p \mathrm{~Pb}$ collisions for $p_{T}>1.5 \mathrm{GeV} / \mathrm{c}$. It reaches a maximum at different $p_{T}$ values depending on $\eta$, following by the fall pointing to unity. This kind of behaviour can be interpreted as Cronin-like [8].

The $R_{p \mathrm{~Pb}}$ is compared with the prediction from phenomenological models: set of nuclear parton distribution functions nPDF fits EPPS16 and the PDF set CT14 for the proton, employed with the fragmentation functions set DSS [9], Color Glass Condensate (CGC, forward region only) [10], perturbative QCD (pQCD, backward configuration) [11]. The first prediction, although with large uncertainties, has the best similarity to data in the case of the forward configuration, albeit it cannot reproduce the $R_{p \mathrm{~Pb}}$ in the backward one.

This measurement represents one of the most precise to date. The total uncertainty is below $5 \%$ for most of the $\left(\eta, p_{T}\right)$ bins. This result could serve as input for future fits of nPDF assuming that nuclear effects in inclusive charge particle production in $p \mathrm{~Pb}$ come predominantly from the modification of nPDF. 


\section{Acknowledgments}

This work was partially supported by the National Research Centre, Poland (NCN), grants No. UMO-2019/35/O/ST2/00546 and MEiN DIR/WK/2017/2020/04-1.

\section{References}

[1] LHCb Collaboration (A Augusto Alves Jr et al.), The LHCb Detector at the LHC, JINST 3 (2008) S08005.

[2] C.A. Salgado et al., Proton-Nucleus Collisions at the LHC: Scientific Opportunities and Requirements, J.Phys.G 39 (2012) 015010.

[3] LHCb Collaboration (R. Aaij et al.), Measurement of prompt charged-particle production in proton-proton collisions at a centre-of-mass energy of $13 \mathrm{TeV}$, hep-ex/2107.10090, submitted to JHEP.

[4] T. Pierog et al., EPOS LHC: Test of collective hadronization with data measured at the CERN Large Hadron Collider, Phys. Rev. C 92 (2015) 034906.

[5] LHCb Collaboration (R. Aaij et al.), Measurement of the nuclear modification factor and prompt charged particle production in $p P b$ and pp collisions at $\sqrt{s_{N N}}=5 \mathrm{TeV}$, hep-ex/2108.13115 submitted to Phys. Rev. Lett.

[6] T. Sjostrand et al., An introduction to PYTHIA 8.2, Comput. Phys. Commun. 191 (2015) 159.

[7] P. Skands, S. Carrazza, and J. Rojo, Tuning PYTHIA 8.1: The Monash 2013 tune, Eur. Phys. J. C74 (2014) 3024.

[8] J. W. Cronin et al., Production of hadrons with large transverse momentum at 200, 300, and $400 \mathrm{GeV}$, Phys. Rev. D 11 (1975) 3105.

[9] K. J. Eskola, P. Paakkinen, H. Paukkunen, and C. A. Salgado, EPPS16: Nuclear parton distributions with LHC data, Eur. Phys. J. C77 (2017) 163.

[10] T. Lappi and H. Mantysaari, Single inclusive particle production at high energy from HERA data to proton-nucleus collisions, Phys. Rev. D88 (2013) 114020.

[11] Z.-B. Kang et al., Multiple scattering effects on heavy meson production in $p+A$ collisions at backward rapidity, Phys. Lett. B740 (2015) 23. 